\title{
Establishing News Credibility using Sentiment Analysis on Twitter
}

\author{
Zareen Sharf ${ }^{1}$ \\ Department of Computer Science \\ SZABIST \\ Karachi, Pakistan
}

\author{
Zakia Jalil ${ }^{2}$, Wajiha Amir $^{3}$ \\ Department of Computer Science \\ International Islamic University \\ Islamabad, Islamabad, Pakistan
}

\author{
Nudrat Siddiqui ${ }^{4}$ \\ Research Scholar \\ Karachi, Pakistan
}

\begin{abstract}
The widespread use of Internet has resulted in a massive number of websites, blogs and forums. People can easily discuss with each other about different topics and products, and can leave reviews to help out others. This automatically leads to a necessity of having a system that may automatically extract opinions from those comments or reviews to perform a strong analysis. So, it may help out businesses to know the opinions of people about their products/services so they can make further improvements. Sentiment Analysis or Opinion Mining is the system that intelligently performs classification of sentiments by extracting those opinions or sentiments from the given text (or comments or reviews). This paper presents a thorough research work carried out on tweets' sentiment analysis. An area-specific analysis is done to determine the polarity of extracted tweets for make an automatic classification that what recent news people have liked or disliked. The research is further extended to perform retweet analysis to describe the re-distribution of reactions on a specific twitter post (or tweet).
\end{abstract}

Keywords-Sentiment analysis; tweets; opinion mining

\section{INTRODUCTION}

The study performed an area-specific sentiment analysis on tweets to extract people's opinions or comments on the recent news. The retweet analysis is also performed in order to describe the re-distribution of reactions on a particular twitter post.

The massive increase of social media (including networking sites, blogs, forums, communities, etc.) on the web has taken a new turn in the form of public opinion. The organizations, businesses and companies now consider public opinion (or feedback) an important aspect in decision making. But filtering out the necessary information from such social sites presents an issue that needs to be resolved. The credibility of social sites is very important to be analyzed as well as the diversity of languages on such social platforms is another problem to deal with. This raises an utmost need of a smart and intelligent analysis to filter out the desired public opinion on a certain issue.

Sentiment Analysis is the solution to the aforementioned problem which is an application of natural language processing (NLP). This analysis system is capable of extracting the opinions or views of public regarding a certain topic. It consists of a system that collects opinions (or feedbacks) on a specific topic from various blogs, social networking sites and reviewing panels. There are two major challenges in sentiment analysis. First one is the opinion words and the second one is the manner in which these opinion words are expressed. The words used in an opinion can be positive (for a specific topic or issue) and negative at the same time for some other issue. Whereas the manner depicts the style or tone in which the words are being used. Either the words are used in a positive sense or the same words are used to taunt something. On the other side, language is another hurdle for many of the smart and accurate sentiment analysis systems. A number of systems have been developed to process English, but an intelligent multilingual system is an utmost requirement to cope with diversity of languages on such social sites.

Due to an emerging trend of communicating and sharing personal opinions, people participate in different events on such sites particularly the ones owned by press media. This participation comes in the form of comments and reviews on a particular news or report. These reviews and comments embody a lot of subjective information. So, some intelligent ways are required to be devised to extract meaningful information from such opinions or comments. In this regard, the NLP systems propose a term Subjective Analysis to handle the mentioned issue. This is an enclosed term that covers sentiments, emotions, opinions and evaluation. The two major approaches that are incorporated for a meticulous and accurate analysis of viewer's responses are Sentiment Analysis and Opinion Mining.

The sentiment or opinion analysis for tweets or comments is far more difficult and challenging than the systems that only collect user's feedback in the form of likes and dislikes. For the tweets (or comments), it might be possible that people have disliked something because of the unnecessary details provided on a certain topic and they are not interested to get into such details. This is the reason sentiment analysis is not merely to check each and every word, rather the system has to be guided to identify and extract what is beneficial for the analysis. It is a fact that tweets contain slang language, various internet writing styles, jokes, icons or commonly used web phrases. All these things make syntax analysis complicated and may lead to wrong classification of tweets. So, this is the major requirement to devise a powerful sentiment analysis system that not only focuses on the extraction of information but also analyses the subjectivity of the tweet. Some of the major challenges for sentiment analysis systems are: 
Named Entity Extraction-This is one of the major challenges. Being existed in every tweet, the named entity extraction refers to the extraction of main idea behind the usage of any name, like the 'Dreams of Mango People' is a term that is used to represent the thoughts and desires of common people.

Information Extraction-Another biggest challenge is the extraction of meaningful information. 'Was the tweet informative or fake?' This is the question that a sentiment analysis system is devised to answer accurately.

Sentiment Determination-It is about determining the sense in which the tweet has been posted. It is either in positive or negative sense. Since sentiments are usually used in a subtle manner that makes it difficult to be analyzed from a single word or sentence. For example:

The private educational institutes are increasing fee by $20 \%$. But these institutes are providing a high-quality of education.

The first part "The private educational institutes are increasing fee by $20 \%$." can be considered as a fact, while the other part is based on a personal opinion. This makes it further clear that identifying a single keyword for subjectivity is not that easy as it seems.

Parsing-In sentiment analysis, this phase is referred to as Semantic Parsing. This actually aims at highlighting the semantic constituents (subject/object, verb/adjective) are identified. It is basically a formal analysis of a sentence performed by the computer.

Anaphora Resolution: This is the phase in which nouns and pronouns are identified. Basically, an anaphora relates an expression to another one preceding it in the discourse. It presents a biggest challenge in Sentiment Analysis. Here the anaphoric expression can be explained by relating them with the context of the whole sentence. Means it can be viewed as a summarization of the context by extracting different sentences. It helps out in Sentiment Analysis by identifying which discourse identities are used repeatedly.

Twitter is one of the widely used social networks that has a massive number of users including politicians, celebrities and companies. The given research focuses on the sentiment analysis performed on Twitter data. The paper is organized as follows:

Section 2 provides a brief background about social media as well as Twitter and tweets. Section 3 gives a thorough literature review. Section 4 deals with the proposed technique that is news credibility using Sentiment Analysis. Section 5 gives implementation details while Section 6 provides performance measures, experimental results. And Section 7 gives conclusion with the recommendations for future work.

\section{BACKGROUND}

With the advent of modern technologies and a gigantic increase of social network communication has made everything online. This is the reason With the advent of modern technologies and a gigantic increase of social network communication has made everything online. This is the reason online communication is getting cheaper. Almost $70 \%$ of the platforms are completely free and this leads to a massive participation of a layman in the form of reviews, comments and discussions regarding news updates, products, services, etc. People from all over the world can give their opinions on such platforms, means the region's restriction is no more. This attracts researchers and analysts who want to analyze such opinions and comments. This is where NLP plays its significant role in which such comments, opinions or tweets are analyzed to extract the useful information with the help of computer programs. This entire process is referred to as 'Opinion Mining'.

The following paper focuses on the Sentiment Analysis carried out on tweets. For this purpose, an area-specific analysis is performed; the user's tweets are monitored on different Pakistani political as well as non-political issues. In order to extract the user's feedback on a specific news, a mechanism (called Sentiment Analysis) is devised that basically analyzes the polarity of those tweets (checking positivity or negativity) to classify either people have liked it or not.

\section{A. Retweet Analysis Description}

First, confirm that you have the correct template for your paper size. This template has been tailored for output on the US-letter paper size. If you are using A4-sized paper, please close this file and download the file "MSW_A4_format".

It refers to the second major phase covered in this paper and plays a significant role in classifying the tweets and their credibility. From different studies, it is found that important news events or updates are retweeted (or shared) more. Similarly, the tweets with negative impact like news related to natural disasters are retweeted more than others. This is why the retweet analysis description is performed to describe the re-distribution of expressions or reactions on a particular tweet or post.

\section{LITERATURE REVIEW}

The major concept behind Sentiment Analysis is to determine the attitude of a user (or a writer or speaker) regarding a specific issue. Or it finds out the overall polarity of a document. Many of the work done in this realm is dependent upon machine learning approach. Due to the huge spread of user generated content through social media and forums, a massive work has been done on the sentiment analysis for a social network. Also referred as Opinion Mining, this process is considered to be highly accurate way to test credibility of any news or topic on social networks. (Denecke, Kerstin, 2008) [1] devised a method that automatically determines the polarity of sentences which are not in the same language. This is called multilingual sentiment analysis. First the text language is determined (if it was other than English) and translated into English in order to carry out sentiment analysis in an easy way. The research also determined the type of document, whether it is subjective or objective. Due to the diversity and complexity involved in human languages, the research came across many difficulties. Moreover, sentiment of text became more crucial, so the whole text was not analyzed. 
Most of the work done on Sentiment Analysis had utilized the approaches of Support Vector Machine (SVM) and other classifiers with binary uni-gram weights. (Paltoglou, Georgios, and Mike Thelwall, 2010) [2] came up with the idea that classification accuracy can be enhanced with information retrieval scheme when more weighting schemes were used. The work modeled a document as an unordered collection of words called a bag of words. The approach came out to be computationally efficient. More sophisticated term weighting functions were adopted from SMART retrieval system to devise a probabilistic model.

(Caladoa, Edgar RochaaAlexandre et al., 2011) [3] had thoroughly discussed about the user contents posted on their respective Twitter profiles as well as what type of people mostly post tweets. In order to carry out this research, they first extracted a list of features. The list comprised of a number of friends, followers, tweets and re-tweets. Moreover, the user's tweeting behavior was tested incorporating feature extraction method. Finally, the public behavior on real time news events was also examined. For this purpose, an algorithm was devised that was used to extract re-tweet chains and timestamp of messages. The algorithm performed user profiling for which the limitation was that this profiling was not enough for analyzing the behaviors of users posting tweets or replying to the tweets regarding news events.

Another work performed on Sentiment Analysis by (Takaoka, Kouichi, and Akiyo Nadamoto, 2011) [4] came up with a new system titled 'Words of Wisdom'. They proposed a system that was based on multi-dimensional sentiments vector, in which two-system approach was utilized. First, a multi-dimensional vector (based on 10 categories of sentiments) was proposed, and then values for these proposed sentiments were calculated. A frequency vector was used to calculate the frequency of sentiments. The results came out to be quite accurate and the authors proposed in future work that distances could also be calculated and adoption for news data could also be incorporated.

Due to a rapid and huge spread of social networking and blogging platforms, researchers are going deeper into this area. Online Opinion Mining is another form of sentiment analysis that has been greatly worked on but it is considered a difficult form of sentiment analysis. (Haddi, Emma et al., 2013) [5] worked on the role of text pre-processing for sentiment analysis and demonstrated how the sentiment analysis can be further significantly improved by using appropriate feature selections and representations. They made use of Support Vector Machine (SVM) and performed a comparison of their accuracies with the accuracies acquired in topic categorization. The research mainly focused on the product reviews (on social blogs) and determined the importance of a product on the basis of those reviews. The supervised learning for feature sections and representations was used as the major technique and the accuracies achieved were in the range of $75 \%$ to $83 \%$.

Going beyond the researchers have shown a great interest in Twitter-specific sentiment analysis. This kind of sentiment analysis is a bit different from the conventional one. Due to the limited character length (up to 140 characters), Twitter messages are full of slangs, short forms, abbreviations. This makes it far difficult from other forms of sentiment analysis. Most of the Twitter sentiment analysis is done using machine learning approach. The two major reasons for using Machine Learning techniques are:

1) A huge amount of Twitter data is available for training datasets.

2) The test data is also available that is user-labeled for sentiment with emoticons, so there is no need of manual annotation of data for training.

The emergence of Web 2.0 has brought improvements in the way people used to perceive Internet. Micro-blogging is one of the most popular Web 2.0 applications that have facilitated users to collaborate, share, discuss and leave their feedbacks on different topics, news and products. Twitter being one of the most popular micro-blogging platforms, has been the hot area of research for many years. (Kontopoulos, Efstratios, et al., 2013) [6] had worked on the same discipline and discussed about the ontology-based techniques for an efficient sentiment analysis of Twitter posts. Their research is divided into two phases: a) creation of domain ontology and b) sentiment analysis on a set of tweets based on the concepts and properties of ontology. This work had utilized FCA (Formal Concept Analysis) which is a mathematical data analysis theory and typically used in knowledge representation and information management. They came across a difficulty in advertising tweets in which an unpleasant ratio was involved.

(Montejo-Ráez, Arturo, et al., 2014) [7] performed Sentiment Polarity Classification on Twitter posts using a novel approach. They extracted a vector of weighted nodes from the graph of WordNet. Then, these weights were used in SentiWordNet in order to compute the final polarity. The method proposed a non-supervised approach that was domain independent. Since Twitter publishes a vast range of information including political, economic, business and more contexts, the scoring of posts is done as per the degree of positive and negative opinions expressed therein. For this purpose, SentiWordNet scores were combined with a random walk analysis of the concepts found in the text over the WordNet graph. Random Walk is an algorithm that was particularly used for mathematical formulization to perform random steps. A graph was also constructed for configuration of the results that demonstrated different parts of WordNet subgraph for the solid terms. The proposed method was intended to calculate the global polarity of Twitter posts by expanding a fewer concepts that were in tweets. The limitation of this work is that they didn't consider whole tweet text form analysis. They also came up with the fact that taking too many concepts will introduce noise in understanding the latent semantic of the text.

(Abbasi, Mohammad-Ali, and Huan Liu., 2013) [8] focused on the social media for information of the upcoming news events in the world. Since people are more interested in getting first hand news, the paper worked on the same issue and proposed a method to measure user credibility in social media. For this purpose, they proposed CredRank algorithm that was solely devised to measure user credibility in social media. It analyzes the users' online behavior to measure the 
said credibility. The proposed methodology worked in the given steps:

- Detect and cluster coordinated (i.e. dependent) users together.

- Weight each cluster on the basis of the cluster's size.

Due to the anonymous and unmonitored mature of the Internet, the user generated content on Twitter might be incredible. This incredibility leads researchers to work on different ways to perform credibility analysis. (Gupta, Aditi, and Ponnurangam Kumaraguru., 2012) [9] worked on this realm and performed credibility analysis on the information contain in a tweet corresponding to fourteen high impact news events of 2011 occurring globally. They conducted research with the help of Regression Analysis through which they identified the important content and source-based features; helpful for predicating the credibility of information contained in a tweet. The ranking of tweets (according to their credibility scores) was performed using the supervised machine learning and feedback approach. The performance of the ranking algorithm was significantly improved when re-ranking strategy was applied. With all the data analyzed, it came out to be known that pn average $30 \%$ of total tweets posted about an event comprised of situational information. While the remaining $14 \%$ contained spam tweets. Whereas only $17 \%$ of the total tweets with situational awareness information were credible. Pseudo Relevance Feedback (PRF) was used for reranking purpose. This technique is also known as Blind Relevance Feedback and one of the most prominent re-ranking techniques used in information retrieval tasks to improve the performance of re-ranking. PRF works by extracting K ranked documents and then re-rank them on the basis of denoted score. The algorithm extracted the most frequent unigrams from the top $\mathrm{K}$ tweets and re-rank them by utilizing the text similarity between those most frequent unigrams and $\mathrm{K}$ tweets. PRF was basically applied to the best set of results that was acquired by previous analysis (that is the ranking results using both message and source. Using the metric BM25, the text similarity between a tweet $\mathrm{T}$ and query set $\mathrm{Q}$ was determined for each event occurred. Around $50 \%$ of tweets on an event are composed of the tweets which were related to the event but didn't provide any useful information. So, it was concluded that a ranking algorithm is based on both the user properties and content. And it turns out to be very effective in determining the credibility of information in these tweets.

(Amiri, Fatemeh et al., 2015) [10] had performed sentiment analysis for Persian text through lexicon-based approach. Since a very little amount of work has been done so far on Persian language, therefore in order to gain insights from different online sites and social media, sentiment analysis was performed. But the researchers came up with a novel approach of incorporating a manually created lexicon that was enriched with sentiment scores, coupled with handcoded grammar rules. The work also addressed some of the Persian language issues including difference between formal and informal writing styles, context sensitivity, complexity due to frequent morphological operations, lexicon intricacy etc. To perform the proposed idea, they first manually collected Persian adjectives, words and expressions from two online resources. After the collection, sentiment annotation was performed in which the collected words were annotated with corresponding sentiment scores. Then, a lexicon based sentiment analysis pipeline was created. This was comprised of steps including: Tokenizer - that splitted text into very simple tokens. Sentence Splitter - that fragmented text into sentences. POS Tagger - It produced a part of speech tag as an annotation for each word or symbol. Gazetteer - This was the basis of the proposed methodology, as each gazetteer entry when appeared in the text, it got marked and was assigned a sentiment score accordingly. After passing the data through this pipeline, the JAPE rules (hand-coded grammar rules) were devised. These rules were formed in two phases - phase I formed word-level rules whereas phase II worked on sentence-level rules. Finally, the Groovy scripting processing resource was utilized through which the number of positive and negative annotations (in a given text piece) were counted and an overall polarity was determined as well. The proposed method yielded around $60-70 \%$ accuracy rates for the initial version of lexicon-based sentiment analysis API. Although, the method did not come up that much efficient as most of the ML based approaches. But it showed value and could be combined with some ML based approach to produce a hybrid system.

(Sharma, Nitesh, et al, 2018) [11] designed a web-based application for performing sentiment analysis of live tweets. Due to a massive use of social media, people use this platform for expressing their opinions on almost every topic. That is why the researchers came up with an idea of building a webbased application that not only performs sentiment analysis on live tweets but also visualizes the measured sentiments associated with keyword (hashtag, words or phrases) of Twitter messages. So, this enables users to measure the sentiment of these messages in terms of geography. The application is designed on the framework 'Flask' using Python programming language. This framework is built in a way that user enters a keyword and application fetches the live tweets (related to the entered keyword), extracts text from each tweet and calculates the user location and sentiment for each tweet and finally plots the results on a map. Flask framework contains an initial configuration file that is used by Views module. This module is responsible for rendering web pages and communicating with API. It intercepts the incoming requests and transfers the control to the back-end layer for processing. As the result is ready, the views module generates web pages. In order to extract tweets, Twitter streaming API is utilized that fetches live tweets corresponding to the entered keyword. Moreover, the attributes (like location, tweet text, followers count, friends count, tweet time) are extracted using the meta data. For extracting the user location, a parser is created that maps user countries to the countries extracted from data. In this regard, there is one additional feature particularly for the US residents that the app generates their state wise plotting on map as well. For sentiment calculation, Python's text-blob library is incorporated. The sentiment score assigned to words range from -1 to +1 and a polarity score of 0 is termed as neutral sentiment. An in-depth analysis of data is also provided in a way that system also calculates the mean and weighted polarity values. Furthermore, the system also calculates the number of tweets from each country and from 
each within state of the USA. Map plotting is done with plotly and Tableau. This application is unique in a sense that it can perform sentiment analysis on live tweets as well as on previously gathered tweets stored in a database. When the system was evaluated using a search string \#Watercrisis, the results were found that a total of 1,164 tweets were extracted related to this hashtag (or keyword) from around the world. And their respective sentiment scores were successfully calculated and visualized on map as well.

(Lauren, Paula, et al., 2018) [12] have conducted a massive research by generating word embeddings from an extreme learning machine for the sentiment analysis and sequence labeling tasks. Word embeddings are basically the low-dimensional distributed word representations for a set of language modeling and feature learning techniques. The words or phrases from the designed vocabulary are mapped on to vectors of real numbers (in low dimensional space). This research focuses on ELM based word embeddings for the sentiment analysis and sequence labeling tasks. There are already different models for generating word embeddings; Word2Vec and Global Vectors (Glo Ve) are the popular of them. In this research, they have also done a comparative study in which ELM based word embeddings are compared with the aforementioned models. Both models use word-pair co-occurrences (also called as Word-Context Matrix) for learning the low-dimensional representation of words. Word2Vec computes this matrix one row at a time while Glo Ve computes the matrix at once and then applies matrix factorization. The first model is known as Predict-based as it performs line-by-line computation, whereas the other one is called as Count-based method because the word pair counts are performed all at once in the first. Their results are comparable, but Word2 Vec takes more time as it needs to train the neural network also, while Glo Ve consumes more memory. On the other hand, Extreme Learning Machine (ELM) is actually a type of feed forward neural network and its efficiency is attributed to the random non-updated hidden layer weights as well as the efficient learning of output layer weights. This study has utilized an Autoencoder architecture based on ELM for its feature learning functionality. Autoencoder itself is a type of neural network that performs feature learning by compressing the input feature vector (in the hidden layer) and then decompressing it back in the output layer. This compression feature is very useful because in generating word embeddings using an ELM, the compressed representation is desired because word embeddings correspond to the low-dimensional representation of WordContext matrix. Furthermore, a recurrent neural network (RNN) is also utilized in this study for the sequence labeling task. An RNN is a feed-forward neural network that contains recurrent connections back to the previous layer. This feature is useful for processing sequential data. They have incorporated Elman RNN for this study. Then for sentiment analysis task (to assess the models of word embeddings), Logistic Regression is applied as a classification algorithm in which the outcome $\mathrm{Y}=1$ means positive result, whereas $\mathrm{Y}=0$ means the outcome is negative. For conducting research, three separate datasets are utilized in which two datasets belong to Sentiment Analysis task and one for Sequence Labeling task. Word embeddings were generated using three models: ELM,
Word2Vec and Glo Ve. Furthermore, Word2Vec includes four models - two from Skip-Gram and two from Continuous Bag of Words (CBOW). The parameters used across all of the models were: minimum word count (means the number of times a word is present in the corpus), word vector dimension size and window context size. Moreover, Word2Vec and Glo Ve required different hyperparamters including Learning rate, Weighting, Iteration and more. Whereas, ELM required only one hyperparamter that is Regularization. For generating ELM word embeddings, the foremost step is to build a vocabulary and its major parameter is minimum word count that finds out how many times a word should appear in the corpus to be included in the vocabulary. Then, frequency counts are rendered for each of the word pairs from the training corpus. The window context size is utilized that determines how far to the left and right each word has to be used for the cooccurrence counts. A weighting scheme is incorporated to get a numerical representation of context, so the closer words are given higher weight and the distant ones are assigned with lower weight values. The word context matrix is a square matrix with the dimensions matching to the size of vocabulary. The square root transformation of the wordcontext matrix with $\mathrm{l}_{2}$ - normalization is done before applying ELM. The word vector dimension size corresponds to the number of neurons in the hidden layer of ELM. For this purpose, the MATLAB ELM Autoencoder source files were used. The study has performed both intrinsic and extrinsic evaluation The intrinsic evaluation means word embeddings are evaluated on the basis of semantic relatedness whereas in extrinsic evaluation the word embeddings are assessed in the downstream NL Task like text categorization. The results on sentiment analysis task are evaluated using Precision, F1 and Recall measures. For the sequence labeling task, Precision and Recall are measured and F1- score is applied utilizing the Precision and Reall equations. In sentiment analysis task, the results of F1-score demonstrate that ELM word embeddings are competitive with Skip-Gram and Glo Ve word embeddings. And for the sequence labeling, Precision, Recall and F1-score are averaged across 20 executions using the test set incorporating the RNN for six models. The experimental results show that CBOWHS model did well overall in comparison of other three Word2Vec models. While the ELM word embeddings show a slightly better average in terms of F1-score as compared to other five models.

(Shirsat, Vishal S et al., 2019) [13] discussed about sentence level sentiment identification by performing research on news articles. The data (of news articles) was extracted from BBC news and sentence-level negation identification was basically applied. Sentiment Analysis is basically categorized as: Document level sentiment analysis in which the polarity of entire document is determined, Sentence-level sentiment analysis in which each sentence is analyzed and polarity is determined. And Aspect-level sentiment analysis that applies analysis on objects and their respective features. This study uses sentence-level sentiment analysis on news articles using Machine Learning Algorithms Support Vector Machine and Naïve Bayes. The proposed methodology consists of five major steps in which the foremost step was to preprocess the dataset in which the irrelevant text (in news articles) like HTML tags, advertisements and scripts are 
removed. So, the data is prepared for text classification in further steps. The practical implementation involves applying Machine Learning algorithms to perform the classification task. Naïve Bayes algorithm is one of them that determines the probability of an occurrence given the probability of another occurrence that has already occurred. Another algorithm that is used in study is Support Vector Machine which is nonprobabilistic algorithm and works well for both sequential and non-sequential data. After preprocessing of data, the next step is to apply Stemming in which the entire document is transformed into lower case to gain uniformity. Stemming is basically the truncation of a word to its root form. After this, the Term Document Matrix is determined, this matrix defines the frequency of terms that appear in the preprocessed dataset. The rows of matrix are collection and columns correspond to the related terms. Then, sentiment score generation is performed with the aid of positive and negative dictionary. For this purpose, each word in the preprocessed dataset is compared to the word in dictionary to determine whether it is positive or negative. Finally, the Naïve Bayes and Support Vector Machine algorithms are applied to perform classification and estimate the accuracy. The experiment was conducted on the five categories of news articles including Entertainment, Business, Politics, Sports and Technology. They utilized Bing Liu dictionary for determining positive and negative words. This dictionary contains 2006 positive and 4783 negative words. The experimental results demonstrated that Naïve Bayes achieved an accuracy of $96.46 \%$ for Entertainment category and the lowest accuracy was $92.63 \%$ that was for Business category. While Support Vector Machine achieved highest accuracy $94.16 \%$ for Politics category and the lowest as $69.01 \%$ in Sports category.

(Iqbal, Farkhund, et al., 2019) [14] came up with a novel approach in which they designed and developed a hybrid framework for performing sentiment analysis. This framework combined ML based algorithm along with lexical database to automatically analyze the online content (including reviews, social media). Then a Genetic algorithm based feature reduction solution is provided through which further accuracy and scalability is achieved. Moreover, they have proposed a cross-disciplinary area of geopolitics to which they have applied the proposed framework as a case study application. It is a complete unique approach in which they tested and experimented the accuracy of the proposed approach by applying it to the topics like terrorism, global conflicts, etc.

\section{Methodology}

This section gives a detailed discussion about the proposed methods for performing the desired task. The main focus of the work is to perform sentiment analysis on the public opinions and reviews on Twitter on the daily news updates. Therefore, the most important part is to determine how people react about a certain news or topic. In this section, the proposed methodology gives an overview about how data is extracted for analysis as well as the method applied to perform extraction. The next phase deals with data pre-processing and how the application shows accuracy in results. In this regard, a tool is proposed that is used to extract data from Twitter and analyzes it according to the applied algorithm.

\section{A. Data Collection}

Data extraction and collection are the major parts of this research. Extraction is actually a time-consuming process. In order to perform this task, first an account was created on Twitter and an application was also created for authentication to extract the required tweets. Twitter API was incorporated to achieve this work. API is an application programming interface, in which a set of protocols and tools for development of different software applications is defined. This API is open source from where the code (related to Twitter data) is extracted and can further be used in different respects of research work.

\section{B. Proposed Architecture}

The proposed architecture of the system is divided into two steps: Sentiment Analysis and Retweet Analysis. Let's analyze both steps here:

Sentiment Analysis Architecture-The proposed architecture of sentiment analysis depicts the entire scenario of sentiment analysis that how it works on the provided data. The major performance measures applied for the manual annotation (during sentiment analysis) are:

Confusion Matrix-Also referred to as contingency table, matching matrix or errors matrix; this measure is applied on both supervised and unsupervised learning systems. This measure is used in order to have a visual overview for the overall performance of the model used in work. Actually, the matrix compares the actual class values with the predicted ones. This way, it analyzes whether the presented model is confused between the two classes or not. The comparison of predicted values with the ground true values gives a clear picture about the proposed model. The matrix is drawn in the form of table shown in Fig. 1.

It is clear from the matrix that there are two classes positive and negative. But they are further divided in boxes and termed as TP, FP, TN and FN

TP corresponds to the true positive terms. These are the values that are positive in actual and the proposed model has also predicted them as positive.

FN means false negative. Means these terms are actually negative but the proposed model did not predict them as negative.

$\mathrm{TN}$ represents the values that are negative in actual and the proposed model has also predicted them to be negative.

FP represents the values that are positive in actual but the proposed model didn't predict them to be positive.

Precision-Precision is basically defined as the ratio of all those instances which are correctly predicted by the classifier as positive. Although, it is a performance measure for binary classification (and we have used multi classification), but it can be used for multi class classification. The formula is defined below:

$\frac{T P}{T P+F P}$ 


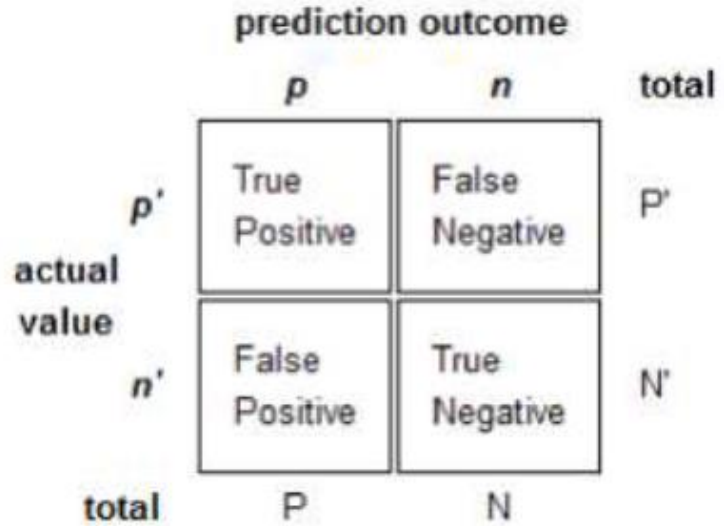

Fig. 1. Confusion Matrix.

Sensitivity-It corresponds to the ability of test for correctly identifying the condition. Also known as Recall, it checks for the strength of classifiers' probability to select instances of a specific class from the whole dataset. It, in actual, corresponds to the true positive rate having the shown formula:

Sensitivity $=\frac{T P}{T P+F N}$

Specificity-It is the measure of the proportion of negative instances (or values) correctly identified from the data set. Means it is opposite of sensitivity. The formula is:

Specificity $=\frac{T N}{T N+F P}$

Kappa Coefficient-This performance measure is very useful in comparing the actual accuracy of a system with random accuracy. As defined by R. Landis and G. Kochh, total accuracy of a system is an observational probability of agreement, whereas random accuracy is a hypothetical expected probability of agreement under an appropriate set of baseline constraints. The Kappa accuracy formula is given here:

Kappa $=\frac{\text { Total accuracy }- \text { Random accuracy }}{1-\text { Total accuracy }}$

Re-tweet Analysis Architecture-A description is given below:

Re-tweet Analysis-The retweet analysis is performed in parallel with sentiment analysis. First, the data is downloaded from Twitter, then source of the original posts is analyzed. These steps are followed by the steps in which re -tweets are checked and counted as well. While analyzing the re-tweets, it is important to be considered that source should not be the same. Means the person who has basically tweeted must not re-tweet it as it comes under an effort to increase the ranking of his post or tweet. For re-tweet count, if it is greater than 35 the news is credible.

Support Vector Machine-Abbreviated as SVM, this is one of the best classifiers which is used for binary classification. Its major purpose is to use the entire data on a high dimension space and try to acquire the maximum margin hyperplane between both data sets.
The Hyperplane-There might be any number of hyperplanes (for SVM) for the specified data points. Moreover, they are further classified into thick and thin hyperplanes. The main objective of SVM classification is to find out a hyperplane that is linearly separable and has the largest margin.

Following constraints are required in order to calculate the hyperplane:

- $\mathrm{x}$ i- the vector that contains the attribute values of all instances of which is the vectors that contains the attribute values of all the instances of $i$.

- $W$ - the vector containing weights of all attributes.

- $b$ - is a real number created for representing the $y$ intercept.

We set the decision boundary on the points that the following equation comes out to be true:

$x \cdot w+b=0$

Suppose, we have two points that lie on the decision boundary. So,

$x \mathrm{a} \cdot w+b=x \mathrm{~b} \cdot w+b=0$

Thus, we can also say that:

$w(x \mathrm{~b}-x \mathrm{a})=0$

where, we know that both these points are parallel to the decision boundary.

The main formula for the hyperplane as generated by $w$ and $b$ is given below:

$f(x i)=x i . w+b$

So, for some point, if $x \mathrm{i} \cdot w+b>0$; it will lie above the hyperplane. And if $x$ i $w+b<0$, it will lie below the hyperplane. We can represent the classes as 1,0 and they can be written in the form

$Y=\{0$, if $x$ i $. w+b>0\}$

$Y=\{1$, if $x \mathrm{i} . w+b<0\}$

These points can be named as Support Vectors.

Distance-The following rule is used to give the distance between margins and the decision boundary:

$\mathrm{D}=\frac{2}{\|w\|}$

We have to estimate the parameters and $b$. w throughout the course of SVM learning.

As mentioned above the key criterion behind SVM classification is to have a correct classification of all points. Fig. 2 presents a visual representation of this.
$\mathrm{x}_{\mathrm{i}} \cdot \mathrm{w}+\mathrm{b} \geq 1$ if $\mathrm{y}=1$
$x_{i} \cdot w+b \geq-1$ if $y=0$ 


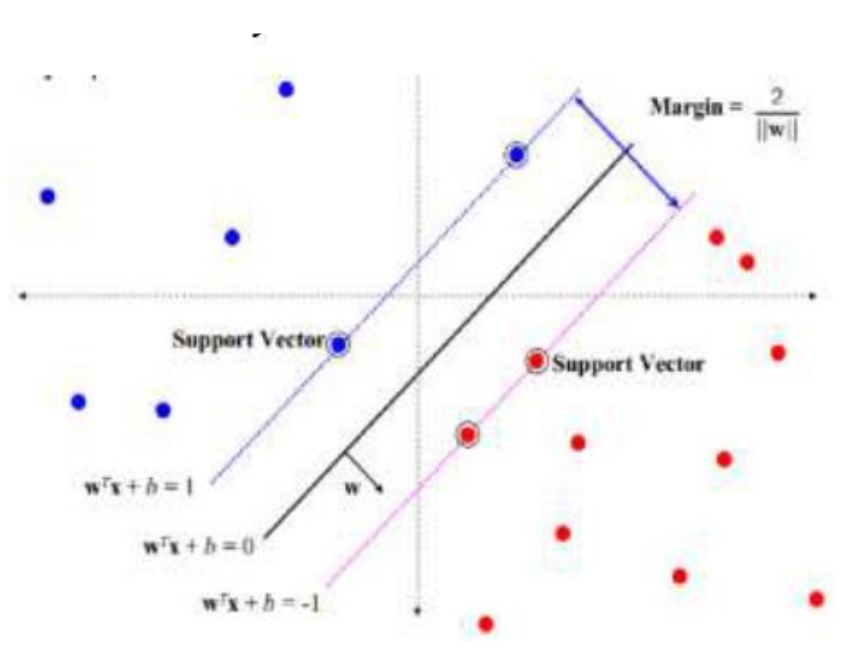

Fig. 2. The SVM Hyperplane.

Furthermore, it is also mentioned above that for SVM's hyperplane, the margin must be the largest. This can be achieved by minimization of the following formula:

$f(w)=\frac{1}{2}\|w\|^{2}$

To carry out minimization, the following constraint must be fulfilled:

$\mathrm{Y}_{\mathrm{i}}\left(\mathrm{w} \cdot \mathrm{x}_{\mathrm{i}}+\mathrm{b} \geq 1\right)$ for $1 \leq \mathrm{i} \leq \mathrm{N}$

Lagrange Multipliers is incorporated to optimize or minimize the constraint. The formula for the multiplier is given here:

$\mathrm{L}(\mathrm{x}, \lambda)=\mathrm{f}(\mathrm{x})+\Sigma_{\mathrm{i}=1 \text { to } \mathrm{m}} \lambda_{\mathrm{i}} \mathrm{g}_{\mathrm{i}}(\mathrm{x})$

The following two steps are required to solve Lagrange Multiplier:

$$
\begin{aligned}
& \frac{\partial L}{\partial x i}=0 \text { for } 1 \leq \mathrm{i} \leq \mathrm{n} \\
& \frac{\partial L}{\partial x i}=0 \text { for } 1 \leq \mathrm{i} \leq \mathrm{m}
\end{aligned}
$$

\section{MODEL IMPLEMENTATION}

This section describes the implementation in detail with the description of tool used and its working. ASP.Net framework is used in this study to perform the task. This is a web application framework developed and marketed by Microsoft. It incorporates programming languages including C\#, VB.Net that enable a programmer to build dynamic and interactive web applications. There are many interactive controls like text boxes, buttons, labels etc. that easily configure and manipulate the code. ASP.Net has extended web forms (as event-driven model) for web applications. As the browser submits a web form to the web server, a markup page is generated in response. All client side activities are sent to the server for full state of processing. It is the sole responsibility of server to process the output in accordance with client's action and triggers the reactions.
Information is stored in two states, Page and Session states. Page State is basically the client's state in which the contents of various input fields are present in the web forms. While the Session State represents the server's state that keeps track of the information globally collected over a session. ASP.NET carries pages to and from the server at runtime and also codes the state of server side components in hidden fields. This is done in order to make server aware of overall running applications and operate it in a two-tiered connected way.

ASP.NET uses and accesses the following data sources:

- Databases (e.g., Access, SQL Server, Oracle, MySQL)

- XML documents

- Business Objects

- Flat files

ASP.NET also hides all the details related to the processes of data access, so a higher level of classes and objects is provided that facilitates the data access. Moreover, ADO,NET is another technology incorporated by ASP.NET that works as a bridge between backend data source and ASP.NET control objects.

Twitter: All the data used in this study is real-time and extracted from Twitter. The whole analysis is performed on the posts, reviews and reactions on the news posts uploaded on Twitter. We have performed the research by restricting the location to Pakistan, so only the news posts belonging to Pakistan were considered. APIS are the downloaded data, they are not used to check location. The data on which experiment is conducted is less as many of the duplicate posts were removed. But in order to check the effect on accuracy by increasing the training data, the most recent Pakistani news are considered.

Test Data: In order to analyze the sentiments of Twitter posts (related to news events), only those tweets are extracted that contain words about news headlines (against a certain threshold value) were collected. These tweets were gathered while considering the location (that is restricted to Pakistan related news updates). All the data was from July 2014 to August 2014.

The second important task is to check the number of replies (known as Re-tweets) on these gathered news based posts. But the source of re-tweet must not be the same as of original tweet (as mentioned earlier). If the source comes out to be same, it will get difficult for finding the credibility of that post because sometimes the same person re-tweets his/her post in order to have an increased re-tweet count.

Total Initial Data: The test data (as described above) is downloaded from Twitter. In order to download the most recent posts, we first checked the trending topics on Twitter's home page. All this data collection was done with the help of APIs. The table (given below) shows complete details including total number of tweets, number of trending topics and their count with positive, negative and neutral tweets. 
Trending Topic: It corresponds to the topics or trends that are currently in search. These trending topics are uploaded daily on Twitter to give the users an overview about the trending news or updates.

Keyword Matching: In order to extract tweets, a specific keyword is given to the application. Then, application starts searching tweets (on the website) related to that entered keyword. As the relevant tweets are found, the application connects with Twitter API to download those tweets. Then, API performs authentication of user with oath keys (that are special keys provided to Twitter users who want to download data from the website). Further analysis is performed after the downloading of data.

Sentiment Analysis: Basically, there are three types of Sentiment Analysis: i) Word based Sentiment Analysis, ii) Sentence based Sentiment Analysis, and iii) Document based Sentiment Analysis. Our study focuses on sentiment analysis of sentence as well as words. We have researched about the reactions, emotions and feelings of people on different news (related to Pakistan). After matching and extracting words related to the feelings, sentiment analysis categorizes the extracted words into three categories: Positive, Negative and Neutral.

Positive Sentiment: The reactions (or comments) on the news (or Twitter post) showing happiness and positivity are categorized as positive sentiments. We have, at the backend, incorporated separate dictionaries with both positive and negative words. Moreover, two languages are considered, English and Urdu. Since, we have performed research on Pakistani news based Twitter posts, so most of the tweets are in Urdu language. The most common positive words are happy, nice, good, well-done, thanks, welcome, I like it and more.

Negative Sentiment: The same procedure (as used in positive sentiments) is applied for negative sentiments. If an extracted post has more negative words than positive and neutral, the overall post is considered negative. The most common negative words are worried, sad, sorrow, angry, crying, bad etc.

Neutral Sentiment: The sentiments that don't show any particular feelings like happy, sad, angry, etc. are the neutral sentiments. It gets difficult to identify the neutral tweets because they contain both positive and negative words. If post contains maximum number of positive words, the polarity is measured positive and if maximum number of negative words, the polarity is negative. Otherwise the post is considered to have neutral polarity.

Polarity Check: To measure polarity of the tweet text, the words are compared with both dictionaries (English and Urdu) in parallel. As the word is found in a dictionary, then polarity is classified, in the second step, as positive, negative or neutral. Then number of words are counted and on the basis of this count, polarity of overall post (i.e. positive, negative or neutral) is assigned.

Training Data: In order to achieve accuracy in results, it is required to train application on specific performance measures. In this regard, human annotation is performed before applying performance measures and it is manually done by checking twitter posts and the sentiments against each trending topic and then applying performance measures.

Manual Annotation on Training Data: Twitter posts are manually labeled and then results achieved are compared with the results provided by the application. The performance measures are used to measure accuracy and all comparisons are made on the basis of these measures. A few examples of positive, negative and neutral tweets which are annotated are provided in Tables I and II.

Classification Results: SVM classifier was utilized to get the desired results. The obtained accuracy is measured to be $95 \%$.

TABLE. I. EXAMPLES OF Positive Posts

\begin{tabular}{|l|l|}
\hline Twitter Posts & Category \\
\hline $\begin{array}{l}\text { \#PTI will make \#KARACHI the city of lights again in Naya } \\
\text { \#Pakistan InshAllah \#PTI4Karachi } \\
\text { \#bekhaufJunooniKarachiwalay }\end{array}$ & Positive \\
\hline $\begin{array}{l}\text { I congratulate @ ImranKhanPTI ; other leaders of PTI on } \\
\text { successful public meeting in Karachi this evening. }\end{array}$ & Positive \\
\hline $\begin{array}{l}\text { Happy Defence Day...Long Live Pakistan @MHaris_ } \\
\text { @ Shiyaikho @jdk_update @ OyeeParkashaayy @ KyunBatow }\end{array}$ & Positive \\
\hline $\begin{array}{l}\text { Proud Soil, Proud Nation with Determined Force...... National } \\
\text { Air Defence Day.......Pakistan Zindabad }\end{array}$ & positive \\
\hline $\begin{array}{l}\text { Allah protect our families in Multan and the surrounding area } \\
\text { from the flood threat@MultanInFlood }\end{array}$ & positive \\
\hline $\begin{array}{l}\text { PML-N \&amp; CM Punjab are famous for progressive work in } \\
\text { Lahore. One big rain disclosed all efficiency in Lahore. }\end{array}$ & positive \\
\hline
\end{tabular}

TABLE. II. EXAMPLES OF NEGATIVE POSTS

\begin{tabular}{|l|l|}
\hline Twitter Posts & Category \\
\hline $\begin{array}{l}\text { Ahmad_Noorani: Geo ISD Real fascist face of PTI. } \\
\text { MQM much better than PTI. Karachi ppl wil reject PTI } \\
\text { terrorists in next elections.. }\end{array}$ & negative \\
\hline $\begin{array}{l}\text { Failed show by PTI in Karachi yesterday, hardly 20K } \\
\text { people came. \#BurnolForPTI }\end{array}$ & negative \\
\hline $\begin{array}{l}\text { After So Much Rain Still 14 Hour LoadShedding in } \\
\text { Lahore camp; in result of that People have attacked Lesco } \\
\text { office Today burned its furniture !! }\end{array}$ & negative \\
\hline $\begin{array}{l}\text { Heavy rain starts again in Lahore with a flood warning } \\
\text { issued. - Bubblews http://t.co/VL8at9ytTT via } \\
\text { @ GoBubblews }\end{array}$ & negative \\
\hline $\begin{array}{l}\text { RT @ ABKool: PMLN brought gullu butts in police to } \\
\text { attack azadi march and CM KP called people from KP to } \\
\text { come protect Khan }\end{array}$ & negative \\
\hline $\begin{array}{l}\text { Atleast five armed militants attacked Astana Fazal in } \\
\text { Sargodha on September 6, Pakistan Defence Day. - } \\
\text { http://t.co/11Beh1ejLQ }\end{array}$ & negative \\
\hline
\end{tabular}




\section{EXPERIMENTAL RESULTS AND DISCUSSION}

This section discusses the experimental results with complete details including tables and graphs. The step by step process is given below:

Keywords selection: This is the step in which user goes to Twitter's homepage and searches for the trending topics. As per the location (of the user) specified, Twitter generates the trending topics. The information is presented in Table III.

Extraction of tweets: The keywords are added through analysis box. Press the create button to add analysis word in the analysis drop down menu. As the Run Analysis button is clicked, the program extracts tweets as per the entered keyword. The tweets are generated in the form of bundles like 300 tweets in one bundle.
Sentiment analysis: Sentiment analysis on extracted tweets is then performed that shows positive, negative or neutral tweets as per the given polarities. For this purpose, this application analyzes the polarity of words in each tweet. And the number of positive and negative words are counted. With higher number of positive words, the polarity is defined to be positive and similarly with negative words. The figure and table shown below specify this step clearly.

Retweet analysis: Retweet Analysis is performed in parallel to show the credibility of results. This analysis works on the concept that if a specific keyword has 35 or more retweets in a particular time interval, that topic is considered to be an interesting one that's why more people are interesting in that update. So, it is labeled as a credible news update. This is presented in Table IV and can be visualized in Fig. 3.

TABLE. III. SENTIMENT ANALYSIS OF TRENDING TOPICS

\begin{tabular}{|c|c|c|c|c|c|}
\hline Keywords & Tweets Count & Negative & Positive & Neutral & Credible/Non-credible \\
\hline Flood-in-Multan & 891 & 519 & 78 & 294 & Credible \\
\hline One Nation Day & 298 & 40 & 113 & 145 & Credible \\
\hline Punjab & 4162 & 3011 & 407 & 744 & Credible \\
\hline Red Zone & 1190 & 323 & 270 & 597 & Credible \\
\hline Defence Day & 595 & 200 & 229 & 166 & Credible \\
\hline Flood in Pakistan & 892 & 831 & 25 & 36 & Credible \\
\hline Operation Zarb E Azb & 594 & 267 & 82 & 245 & Credible \\
\hline Civil Disobedience & 1784 & 756 & 343 & 685 & Credible \\
\hline Umar Akmal & 981 & 181 & 219 & 491 & Credible \\
\hline Rain in Lahore & 298 & 257 & 9 & 32 & Credible \\
\hline iPhone 6 & 826 & 199 & 178 & 449 & Credible \\
\hline Tsunami in Karachi & 298 & 282 & 7 & 9 & Credible \\
\hline Azadi March & 893 & 424 & 175 & 294 & Credible \\
\hline PMLN & 1188 & 523 & 199 & 466 & Credible \\
\hline Free-and-fair-Election & 398 & 77 & 268 & 53 & Credible \\
\hline PTI in Karachi & 1785 & 384 & 661 & 740 & Credible \\
\hline
\end{tabular}

TABLE. IV. RETWEET ANALYSIS CREDIBILITY TABLE

\begin{tabular}{|l|l|l|l|l|l|l|}
\hline SR.\# & Id analysis & text & retweet_start_Date & retweet_end_Date & retweet_result & Retweet score \\
\hline 1 & 1184785917 & Flood-in-Multan & $9 / 14 / 20140: 00$ & $9 / 14 / 2014$ & credible & 81 \\
\hline 2 & 1230244833 & One Nation Day & $9 / 13 / 20140: 00$ & $9 / 13 / 2014$ & credible & 38 \\
\hline 3 & 1283045768 & Punjab & $9 / 13 / 20140: 00$ & $9 / 13 / 2014$ & credible & 67 \\
\hline 4 & 1387115085 & Red Zone & $9 / 13 / 20140: 00$ & $9 / 13 / 2014$ & credible & 35 \\
\hline 5 & 1391593695 & Defence Day & $9 / 12 / 20140: 00$ & $9 / 12 / 2014$ & credible & 52 \\
\hline 6 & 2030857401 & Flood in Pakistan & $9 / 12 / 20140: 00$ & $9 / 12 / 2014$ & credible & 60 \\
\hline 7 & 2065829408 & Operation Zarb E Azb & $9 / 13 / 20140: 00$ & $9 / 13 / 2014$ & credible & 37 \\
\hline 8 & 2138605727 & Civil Disobedience & $9 / 13 / 20140: 00$ & $9 / 13 / 2014$ & credible & 65 \\
\hline 9 & 254201070 & Umar Akmal & $9 / 14 / 20140: 00$ & $9 / 14 / 2014$ & credible & 53 \\
\hline 10 & 289536224 & Pak Army in Islamabad & $9 / 12 / 20140: 00$ & $9 / 12 / 2014$ & NULL & NULL \\
\hline 11 & 366838366 & Rain in Lahore & $9 / 13 / 20140: 00$ & $9 / 13 / 2014$ & credible & 65 \\
\hline 12 & 474851049 & iPhone 6 & $9 / 13 / 20140: 00$ & $9 / 13 / 2014$ & credible & 59 \\
\hline 13 & 553443382 & Tsunami in karachi & $9 / 12 / 20140: 00$ & $9 / 12 / 2014$ & credible & 44 \\
\hline 14 & 677447376 & Azadi March & $9 / 13 / 20140: 00$ & $9 / 13 / 2014$ & credible & 62 \\
\hline 15 & 973193056 & PMLN & $9 / 13 / 20140: 00$ & $9 / 13 / 2014$ & credible & 58 \\
\hline 16 & 1738732573 & PTI-in-karachi & $9 / 23 / 20140: 00$ & $9 / 23 / 2014$ & credible & 71 \\
\hline
\end{tabular}




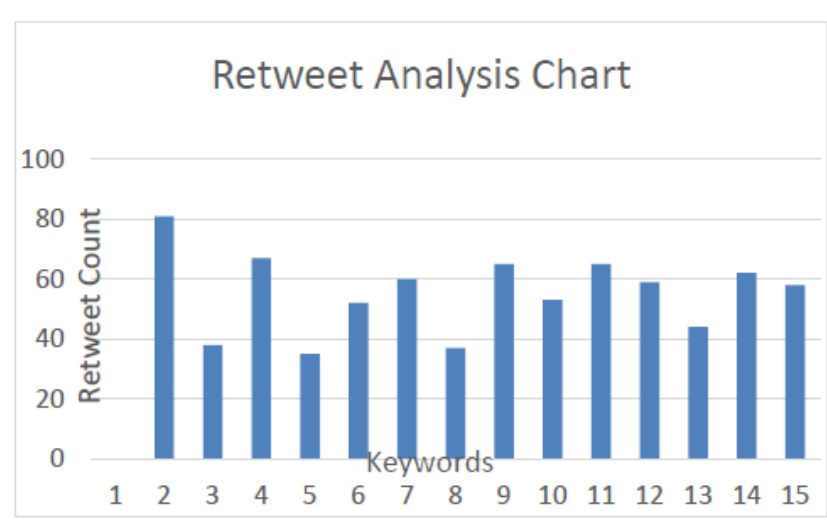

Fig. 3. Retweet Chart.

Output Result-In order to check performance, the performance measures used were precision and recall. A detailed account of the experiment conducted and results achieved is presented in the sections to follow.

Experimental Setup-Here the experimental results are shown in the form of tables according to the applied performance measures. Table $\mathrm{V}$ shows the dataset that we have used. This dataset comprises of the extracted tweets from Twitter and analysis was made on the recent and trending topics.

Now some of the extracted topics and their relevant results are discussed.

Topic $=$ "Red Zone'-This topic was considered negative news before running analysis. The results acquired after running application over 1400 extracted tweets also prove that the news update is negative. The accuracy of the result was checked using performance measures and their respective values are given Table VI. Statistics related to confusion matrix are presented in Fig. 4.

Overall Accuracy: $81.71 \%$

Kappa coefficient: 0.628

Sensitivity: $80.60 \%$

Specificity: $82.24 \%$

Values of chart area:

True Positive $=108$, True Negative $=125$, False Positive $=27$, False Negative $=25$

Topic $=$ "Defense Day"-Obviously, this news update is positive and our application also generated the same result by achieving positive polarity for this news update. Among 975 extracted tweets, there were 375 positive ones, 252 negative and rest of the tweets were neutral. After the results were acquired, the performance measures were applied to check the accuracy and are presented in Table VII. Statistics related to confusion matrix are presented in Fig. 5.

Overall Accuracy: $81.65 \%$

Kappa coefficient: 0.623

Sensitivity: $86.67 \%$

Specificity: $76.88 \%$

Values of chart area:

True Positive $=195$, True Negative $=130$, False Positive $=37$, False Negative $=36$
TABLE. V. EXPERIMENTAL SETUP

\begin{tabular}{|l|l|}
\hline Dataset details \\
\hline Attributes no. & Five classes \\
\hline Characteristics of attribute & Integers \\
\hline Missing values & Nil \\
\hline Predicted variables & Positives and negative tweets \\
\hline
\end{tabular}

TABLE. VI. Precision and Recall Values (Red Zone)

\begin{tabular}{|l|l|l|l|l|}
\hline & Class 1 & Class 2 & $\begin{array}{l}\text { Classificati } \\
\text { on overall }\end{array}$ & $\begin{array}{l}\text { Procedure } \\
\text { Accuracy(precision) }\end{array}$ \\
\hline Class 1 & 108 & 27 & 135 & $80 \%$ \\
\hline Class 2 & 26 & 125 & 151 & $82.78 \%$ \\
\hline Truth overall & 134 & 152 & 286 & \\
\hline $\begin{array}{l}\text { User accuracy } \\
\text { (recall) }\end{array}$ & $\begin{array}{l}80.59 \\
\%\end{array}$ & $\begin{array}{l}82.23 \\
\%\end{array}$ & & \\
\hline
\end{tabular}

\section{Red Zone}

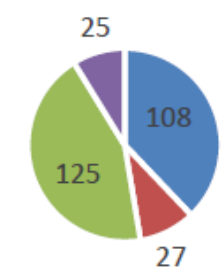

- $\mathrm{TP}=\mathrm{FP}=\mathrm{TN}=\mathrm{FN}$

Fig. 4. Confusion Matrix Values (Red Zone).

TABLE. VII. PRECISION AND ReCAll VAlues (DEFENSE DAY)

\begin{tabular}{|l|l|l|l|l|}
\hline & Class 1 & Class 2 & $\begin{array}{l}\text { Classification } \\
\text { overall }\end{array}$ & $\begin{array}{l}\text { Procedure } \\
\text { Accuracy(precision) }\end{array}$ \\
\hline Class 1 & 195 & 37 & 232 & $80.05 \%$ \\
\hline Class 2 & 36 & 130 & 166 & $78.31 \%$ \\
\hline Truth overall & 231 & 167 & 398 & \\
\hline $\begin{array}{l}\text { User accuracy } \\
\text { (recall) }\end{array}$ & $84.41 \%$ & $77.84 \%$ & & \\
\hline
\end{tabular}

\section{Defence Day}

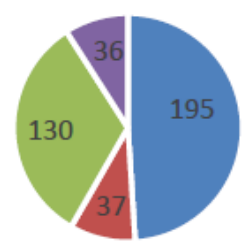

$$
\text { - } \mathrm{TP}=\mathrm{FP}=\mathrm{TN}=\mathrm{FN}
$$

Fig. 5. Confusion Matrix Values(Defense Day). 
Topic $=$ "Rain in Lahore"-The polarity found for this news update was negative. In the month of September, heavy rain occurred that was quite destructive for Lahore and the cities around Punjab. The application gave 298 tweets in which 257 were negative tweets and merely 9 were positive ones. The accuracy results (using performance measures) are provided in Table VIII. Statistics related to confusion matrix are presented in Fig. 6.

Overall Accuracy: $81.57 \%$

Kappa coefficient: 0.513

Sensitivity: $54.55 \%$

Specificity: $92.59 \%$

True Positive $=30$, True Negative $=125$, False Positive $=10$, False Negative $=25$

The overall accuracy achieved for each of the extracted topics depicts the authenticity of the proposed system and the potential it has to get enhanced for more advanced results in future.

TABLE. VIII. PRecision AND ReCALl VAlues (RAIN IN LAHORE)

\begin{tabular}{|l|l|l|l|l|}
\hline & Class 1 & Class 2 & $\begin{array}{l}\text { Classification } \\
\text { overall }\end{array}$ & $\begin{array}{l}\text { Procedure } \\
\text { Accuracy(precision) }\end{array}$ \\
\hline Class 1 & 30 & 10 & 40 & $75 \%$ \\
\hline Class 2 & 25 & 125 & 150 & $83.33 \%$ \\
\hline Truth overall & 55 & 135 & 190 & \\
\hline $\begin{array}{l}\text { User accuracy } \\
\text { (recall) }\end{array}$ & $54.54 \%$ & $92.59 \%$ & \multicolumn{3}{|l}{} \\
\hline
\end{tabular}

\section{Rain in Lahore}

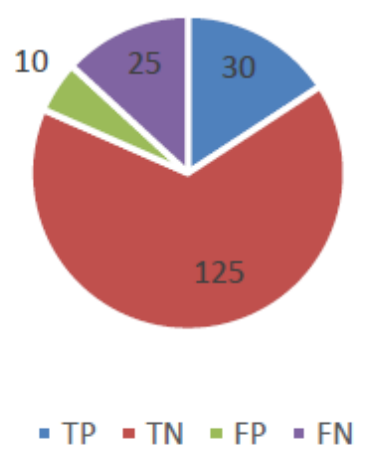

Fig. 6. Confusion Matrix Values (Rain in Lahore).

\section{VII.CONCLUSION}

The research is aimed at solving a practical problem of Sentiment Analysis of Twitter posts related to news updates. We have presented the background, related literature review as well as possible approaches, techniques, features and assumptions for Sentiment Analysis about news events. The data collection process is also thoroughly specified that we have collected the required data with the help of Twitter APIs.
After collecting the tweets related to news updates, they were analyzed to determine their type as per their respective polarities. The chances of noisy data were reduced by downloading the tweets through keyword matching process.

One point is to be cleared that the tweets extracted are all related to the trending news events means the topics that are in great discussion and appear on Twitter's homepage on a daily basis. The reviews or reactions on such tweets are used to determine the polarity as well as credibility of the news updates. It was found during the study that sentiment analysis of tweets can be performed independently without concern of their context. But feature extraction is the crucial element that matters a lot. In this regard, uni-gram and bi-gram proved to be the better features than other ones for performing reliable sentiment analysis.

In the next phase, the re-tweet analysis was another challenging task to be performed. By setting a threshold value $>=35$, the approach worked quite better. This analysis was performed in parallel and credibility of the news events was proved on the basis of re-tweet analysis. If a particular keyword has 35 or more retweets, the topic was considered to be interesting. And more people were found to be taking interest in that topic. This further proved that news is credible.

There were some challenges faced during this study. The collection of tweets related to news updates was quite a challenging and difficult task. After overcoming this challenge, we came across with another challenge that was in the form of manual annotation. In manual annotation, it is difficult to decide whether a given tweet is positive, negative or neutral. Since the tweets are related to trending news events and there is always a possibility that tweet is dependent on the context that makes it further difficult. In this regard, a subjective approach is required because annotation is highly subjective. The limit of 140 characters in a tweet also brings some difficulties, as people find difficulty in expressing their views or opinions in such a restricted limit. So, they use abbreviations, short forms and slangs that make sentiment analysis further challenging.

More future work and studies can be done in this realm. By proposing more complex algorithms and utilizing appropriate measures, a more scalable approach can be proposed. The advanced techniques can be used to enhance the work for resolving issues like detecting fraudulent or criminal activities on social media. Moreover, we focused on English and Urdu languages while ignoring the slang terms. The future work may incorporate the technique that may be capable of analyzing the slang terms as well.

\section{REFERENCES}

[1] Denecke, Kerstin, "Using sentiwordnet for multilingual sentiment analysis." IEEE 24th International Conference on Data Engineering Workshop, 2008.

[2] Paltoglou, Georgios, and Mike Thelwall, "A study of information retrieval weighting schemes for sentiment analysis" Proceedings of the 48th annual meeting of the association for computational linguistics. Association for Computational Linguistics, 2010.

[3] Caladoa, Edgar RochaaAlexandre P. Franciscoa Pável, and H. SofiaPintoa." User profiling on Twitter", 2011. 
[4] Takaoka, Kouichi, and Akiyo NadamotoK. Elissa, "Title of paper if known," unpublished." Words-of-wisdom search based on multidimensional sentiment vector", Proceedings of the 13th International Conference on Information Integration and Web-based Applications and Services. ACM, 2011.

[5] Haddi, Emma, Xiaohui Liu, and Yong Shi." The role of text preprocessing in sentiment analysis.”, Procedia Computer Science, 2013.

[6] Kontopoulos, Efstratios and Berberidis, Christos and Dergiades, Theologos and Bassiliades, Nick." Ontology-based sentiment analysis of twitter posts.", Expert systems with applications, 2013.

[7] Montejo-Raez, Arturo and Martinez-C'amara, Eugenio and MartinValdivia, M Teresa and Urena-Lopez, L Alfonso." Ranked wordnet graph for sentiment polarity classification in twitter", Computer Speech \& Languag, 2014.

[8] Abbasi, Mohammad-Ali, and Huan Liu." Measuring user credibility in social media.", International Conference on Social Computing, Behavioral-Cultural Modeling, and Prediction., 2013.

[9] Gupta, Aditi, and Ponnurangam Kumaraguru." Credibility ranking of tweets during high impact events.", Proceedings of the 1st workshop on privacy and security in online social media. ACM. 2012.
[10] Amiri, Fatemeh, Simon Scerri, and Mohammadhassan Khodashahi." Lexicon-based sentiment analysis for Persian Text.". Proceedings of the International Conference Recent Advances in Natural Language Processing. 2015.

[11] Sharma, Nitesh and Pabreja, Rachit and Yaqub, Ussama and Atluri, Vijayalakshmi and Chun, Soon and Vaidya, Jaideep." Web-based application for sentiment analysis of live tweets.". Proceedings of the 19th Annual International Conference on Digital Government Research: Governance in the Data Age..2018.

[12] Lauren, Paula and Qu, Guangzhi and Yang, Jucheng and Watta, Paul and Huang, Guang-Bin and Lendasse, Amaury." Generating word embeddings from an extreme learning machine for sentiment analysis and sequence labeling tasks.". Cognitive Computation.2018.

[13] Shirsat, Vishal S., Rajkumar S. Jagdale, and Sachin N. Deshmukh. "Sentence Level Sentiment Identification and Calculation from News Articles Using Machine Learning Techniques." Computing, Communication and Signal Processing. Springer, Singapore, 2019. 371376.

[14] Iqbal, Farkhund and Hashmi, Jahanzeb Maqbool and Fung, Benjamin CM and Batool, Rabia and Khattak, Asad Masood and Aleem, Saiqa and Hung, Patrick CK.” A Hybrid Framework for Sentiment Analysis using Genetic Algorithm based Feature Reduction.”.IEEE Access.2019. 